\title{
SETTLING AND RISING VELOCITY OF A SPHERICAL PARTICLE IN HOMOGENEOUS TURBULENCE
}

\author{
Kiyosi KAWANISI ${ }^{1}$, Peter NIELSEN ${ }^{2}$ and Qing-Chuan ZENG ${ }^{3}$ \\ ${ }^{1}$ Member of JSCE, Dr. of Eng., Assoc. Professor, Dept. of Civil Engineering, Hiroshima University (1-4- \\ 1, Kagamiyama, Higashi-Hiroshima 739-8527, Japan) \\ ${ }^{2}$ Ph. D., Assoc. Professor, Dept. of Civil Engineering, The University of Queensland (St. Lucia, Queens- \\ land 4072, Australia) \\ ${ }^{3} \mathrm{Ph}$. D. student, Dept. of Civil Engineering, The University of Queensland (St. Lucia, Queensland 4072, \\ Australia)
}

The average settling and rising velocity in homogeneous turbulent fields of a spherical particle have been examined. The velocity field of homogeneous isotropic turbulence is simulated by Kraichnan's technique. Both experimental and numerical results are shown. From experimental and numerical results, it is found that both light and heavy particles tend to be delayed by relatively weak turbulence. The results also show an increase in the average settling velocity when turbulence intensity is relatively strong. In the experiments, the rising velocity for a light particle decreases greatly with increasing relative turbulence intensity. An decrease by a factor of 0.05 is observed in the grid turbulence. The increases and decreases in the simulation are small in comparison with those in the experiments.

Key Wards: Settling and rising velocity, kinematic simulation, turbulent field, grid turbulence, vortex trapping, fast track

\section{INTRODUCTION}

A knowledge of the mean settling or rising velocity of particles in turbulent flows is important to many problems such as the transport of particulate pollutants, suspension of phytoplankton and sediment, the behavior of aerosol particles and bubbles. Murray ${ }^{1)}$ examined the settling velocities of heavy particles $\left(\rho_{f}<\rho_{p} ; \rho_{f}\right.$ : fluid density, $\rho_{p}$ : particle density) in a turbulence field generated by oscillation of grids in still water. Murray's data ${ }^{1)}$ show that the average settling velocity of a particle in turbulent water can be considerably reduced below its terminal settling velocity in still water though there were the apparent increases in the settling velocity for a quasi-neutral particle.

Nielsen ${ }^{2}$ monitored the settling velocities of a heavy particle in a turbulence tank with oscillating grid plates. He found that the reduction and increase of settling velocity were due to the ratio of the turbulent intensity, $\sigma$, and terminal settling velocity, $w_{0}$, i.e. heavy particles to be slowed by $20-40 \%$ in relatively weak grid turbulence $\sigma / w_{0}<4$, while the settling velocity can be increased considerably in stronger turbulence $\sigma / w_{0}>4$.

Yamasaka \& Ikeda ${ }^{3)}$ and Gotoh et al. ${ }^{4)}$ examined the settling velocities of a heavy particle under vertical fluid oscillations with various frequencies. They found that there were only very small reductions in the settling velocity by the vertical fluid oscillations. However, significant mechanisms of the reductions cannot be expressed in their analysises based on a onedimensional equation of motion. 
There are four theories that predict effects of turbulence on the average settling (or rising) velocity of a particle. The nonlinear drag, vortex trapping and loitering effect lead to the reduction in settling velocity. Conversely, the fast tracks along the vortex boundaries result in an increase of settling velocity.

It was shown experimentally by Tooby et $\left.a l . .^{5}\right)$ that forced vortices with horizontal axes can trap bubbles and heavy particles and thus eliminate their settling velocity completely. The trapping effect is not peculiar to the somewhat artificial forced vortex: it is possible in most vortices including the Rankine vortex and irrotational vortex ${ }^{6)}$.

The fast tracking between vortices was described by Maxey \& Corrsin ${ }^{7)}$ for particles settling through a computer-generated periodic array of steady vortices. They just showed that the heavy particles were gathered in the border areas between vortices. Nielsen ${ }^{2}$ identified these areas as "fast tracks". The same effect was also found by Maxey $^{8)}$ and Wang \& Maxey ${ }^{9)}$ in a simulated turbulent field, though there were only very small increases ( $\leqslant 10 \%$ ).

The experimental data for settling velocity are sparse. Furthermore, few experimental data for the rising velocity of a light particle are available.

In the present study, the settling and rising velocities for heavy and light particles are monitored in a turbulence tank. Moreover, the motion of heavy and light particle are simulated in threedimensional turbulent velocity fields generated by Kraichnan's technique ${ }^{10)}$.

\section{MATERIALS AND METHODS}

\section{(1) Experiments}

The settling or rising velocities of five types of quasi-spherical particles were monitored in the same tank as that of Nielsen's experiment ${ }^{2}$. The tank has a base $0.5 \mathrm{~m}$ square and a height of $0.7 \mathrm{~m}$. The turbulence was generated by two connected horizontal grids spaced $0.5 \mathrm{~m}$ apart.

Between a motor and the grid is a drive disk fixed to the motor shaft, a linkage bar attached to the disk, and a drive rod fastened to the linkage bar. The grid elements are perforated with holes $75 \mathrm{~mm}$ in diameter. The distance between the centers of two neighboring holes is $150 \mathrm{~mm}$. The grids oscillated vertically in filtered water at frequencies of $0.32-1.45 \mathrm{~Hz}$ with a fixed excursion of $50 \mathrm{~mm}$. Two synchronized CCD-cameras,
Table 1 Summary of experimental condition.

\begin{tabular}{cccccc}
\hline \hline & $\begin{array}{c}\text { Grid } \\
\text { Frequency } \\
(\mathrm{Hz})\end{array}$ & $\begin{array}{c}\text { Particle } \\
\text { Diameter } \\
(\mathrm{mm})\end{array}$ & $\begin{array}{c}\text { Terminal } \\
w_{0} \\
(\mathrm{~mm} / \mathrm{s})\end{array}$ & $\begin{array}{c}\text { Average } \\
\bar{w}_{p} \\
(\mathrm{~mm} / \mathrm{s})\end{array}$ & $\begin{array}{c}\text { Standard } \\
\text { Deviation } \\
\sigma_{p} \\
(\mathrm{~mm} / \mathrm{s})\end{array}$ \\
\hline $\mathrm{a}$ & 1.16 & 1.84 & 4.49 & 0.21 & 43.96 \\
\hline $\mathrm{b}$ & 0.32 & 1.81 & 13.01 & 3.21 & 16.81 \\
& 0.48 & 1.81 & 13.01 & 3.09 & 25.69 \\
& 1.16 & 1.81 & 13.01 & 2.26 & 48.02 \\
\hline $\mathrm{c}$ & 0.32 & 1.85 & 38.47 & 13.42 & 16.59 \\
& 1.16 & 1.85 & 38.47 & 3.22 & 42.78 \\
\hline & 0.32 & 1.76 & 1.776 & 0.197 & 17.30 \\
$\mathrm{~d}$ & 0.48 & 1.76 & 1.776 & 0.091 & 21.46 \\
& 1.16 & 1.76 & 1.776 & 0.081 & 48.24 \\
\hline $\mathrm{e}$ & 1.22 & 1.51 & -4.457 & -6.297 & 46.22 \\
& 1.45 & 1.51 & -4.457 & -14.7 & 51.79 \\
\hline
\end{tabular}

looking from perpendicular directions, were used for complete specification of particle positions in three dimensions.

A summary of the experimental results is given in Table 1. The last column shows standard deviations of the instantaneous vertical velocity of particles.

\section{(2) Simulation of turbulence and particle motion}

In order to generate homogeneous isotropic turbulence fields, we used a technique known as Kinematic Simulation (KS) ${ }^{10)}$, which was introduced by Kraichnan. In KS there is no restriction on the Reynolds number. So KS gives one the possibility to study a wide range of values of integral length scales and velocity fluctuations. KS technique can be reviewed as follows.

A turbulent velocity field is represented as follows:

$$
\begin{aligned}
\mathbf{u}(\mathbf{x}, t)=\sum_{n=1}^{N}\left[\tilde{\mathbf{a}}_{n} \cos \left(\mathbf{k}_{n} \cdot \mathbf{x}+\omega_{n} t\right)\right. \\
\left.+\tilde{\mathbf{b}}_{n} \sin \left(\mathbf{k}_{n} \cdot \mathbf{x}+\omega_{n} t\right)\right]
\end{aligned}
$$

where

$$
\tilde{\mathbf{a}}_{n}=\mathbf{a}_{n} \times \frac{\mathbf{k}_{n}}{\left|\mathbf{k}_{n}\right|}, \quad \tilde{\mathbf{b}}_{n}=\mathbf{b}_{n} \times \frac{\mathbf{k}_{n}}{\left|\mathbf{k}_{n}\right|},
$$

which insures incompressibility $\nabla \cdot \mathbf{u}=0$. The number of modes $N$ is large, typically 200 . The random wave vectors $\mathbf{k}_{n}$ were selected using a way by Fung et $a l .{ }^{11)}$ Here, the direction of each wave vector is (uniformly) random. A uniformly random vector $(x, y, z)$ of unit length is obtained as follows: a random coordinate $z$ in the interval $[-1,1]$ and an angle $\theta$ in the interval $[0,2 \pi]$ are picked from a uniformly random distribution; the coordinates $x$ and $y$ are 
$x=\sqrt{1-z^{2}} \cos \theta, y=\sqrt{1-z^{2}} \sin \theta$. The value of each length $k_{n}=|\mathbf{k}|_{n}$ is determined from a spatial energy-spectrum function $E(k)$ :

$$
\int_{0}^{k_{n}} E(k) d k=\frac{3}{2}\left(n-\frac{1}{2}\right) u_{0}^{2} / N,
$$

with

$$
\frac{3}{2} u_{0}^{2}=\int_{0}^{\infty} E(k) d k .
$$

The random (independent) vector coefficients $\mathbf{a}_{n}$ and $\mathbf{b}_{n}$ are determined by choosing a direction uniformly random and choosing a length from a Gaussian distribution with zero mean, and standard deviation $\overline{\left|\mathbf{a}_{n}\right|}$.

Finally, the frequencies $\omega_{n}$ are chosen randomly from a Gaussian distribution with zero mean, and standard deviation

$$
\sqrt{\overline{\omega_{n}^{2}}}=a_{*} u_{0} k_{n}
$$

This implies that the energy-spectrum function $\mathcal{E}(k, \omega)$ has the form

$$
\begin{gathered}
\mathcal{E}(k, \omega)=E(k) \operatorname{cxp}\left[-\frac{\omega^{2}}{2\left(a_{*} k u_{0}\right)^{2}}\right] \\
/\left(\sqrt{2 \pi}\left(a_{*} k u_{0}\right)\right) .
\end{gathered}
$$

The direct numerical simulations of Hunt et al. ${ }^{12)}$ show that a good approximation for $\mathcal{E}(k, \omega)$ is Eq.(6) with $a_{*} \simeq 0.40$ for $k L_{11} \leq 10$, and $a_{*} \simeq$ 0.51 for $k L_{11} \geq 15$, where $L_{11}$ is the longitudinal integral scale.

The only quantity to be specified in the simulation technique described above is the spatial energy-spectrum function $E(k)$. In the present study, the simulations have been performed with the von Kármán-Pao spectrum function, which was found by Helland et al. ${ }^{13)}$ The von KármánPao spectrum function is expressed as follows:

$$
\begin{aligned}
E(k)= & \alpha \frac{\nu^{2}}{\eta}\left(\frac{L}{\eta}\right)^{\frac{5}{3}} \frac{(k L)^{4}}{\left.[1+k L)^{2}\right]^{\frac{17}{6}}} \\
& \exp \left(-\frac{3}{2} \beta(k \eta)^{\frac{4}{3}}\right)
\end{aligned}
$$

where $L$ is the 'energy' scale, $\alpha$ is the Kolmogorov constant and $\eta$ is the Kolmogorov length scale. The parameters $\alpha$ and $\beta$ become equal at large Reynolds number.

The spectrum is fully determined by a specification of the turbulent intensity $u_{0}$ and one of the length scales $L_{11}$ or the Taylor microscale. Kolmogorov microscale is determined from the empirical relationship ${ }^{14)}$ :

$$
\frac{\eta}{L_{11}} \simeq 1.0\left(\frac{u_{0} L_{11}}{\nu}\right)^{-\frac{3}{4}}
$$

The following equation of motion of a spherical particle is adopted in the present simulation.

$$
\begin{aligned}
\left(s+\frac{1}{2}\right) \frac{d \mathbf{u}_{p}}{d t}= & (s-1) \mathbf{g}+\frac{3}{2} \frac{\mathrm{Du}}{\mathrm{D} t} \\
& -\frac{3}{4} \frac{C_{D}}{d}\left|\mathbf{u}_{p}-\mathbf{u}\right|\left(\mathbf{u}_{p}-\mathbf{u}\right) \\
& -\frac{1}{2}\left(\mathbf{u}_{p}-\mathbf{u}\right) \times \Omega,
\end{aligned}
$$

where $\mathbf{u}_{p}(t), \mathbf{u}(\mathbf{x}(t), t), \boldsymbol{\Omega}(\mathbf{x}(t), t)$, and $s$ are the particle velocity, the fluid velocity and vorticity at the position of the particle, and the specific density of particle respectively. The drag coefficient $C_{D}$ is calculated using an empirical expression by Clift et al. ${ }^{15)}$ :

$$
C_{D}=\frac{24}{R e}\left(1+0.1935 R e^{0.6305}\right) .
$$

The last term in Eq. (9) is the lift force: it arises from the presence of vorticity in the ambient fluid motion $^{16)}$.

Given a fluid velocity field Eq. (9) can be integrated numerically. By averaging over many of such 'realizations of the flow', averaged quantities like the mean settling or rising velocity can then be obtained. In the present simulations, the Bulirsch-Stoer method ${ }^{17)}$ with adaptive stepsize control is used to integrate Eq. (9).

\section{RESULTS AND DISCUSSION}

\section{(1) Experimental data}

Our results in terms of relative settling (or rising) velocity $\bar{w}_{p} / w_{0}$ vs. relative turbulence intensity $\sigma_{p} / w_{0}$ are shown in Fig. 1 together Murray's results. The particle turbulence-intensity $\sigma_{p}$ is taken as the standard deviation of the vertical velocity of a particle along its path. The particles in Murray's experiments were approximately spherical in shape, and their diameter was kept constant at $2 \mathrm{~mm}$. Variation of the particle density gave heavy-particle terminal fall velocities of $2,10,20,30$, and $40 \mathrm{~mm} / \mathrm{s}$.

Fig. 1 indicates that heavy particles $\left(\rho_{f}<\right.$ $\left.\rho_{p}\right)$ tend to be slowed by $20-40 \%$ in relatively weak grid turbulence. Conversely, the settling velocity can be increased considerably in strong turbulence. It seems that the turning point from reduction to increase is $\sigma_{p} / w_{0} \approx 10$. 


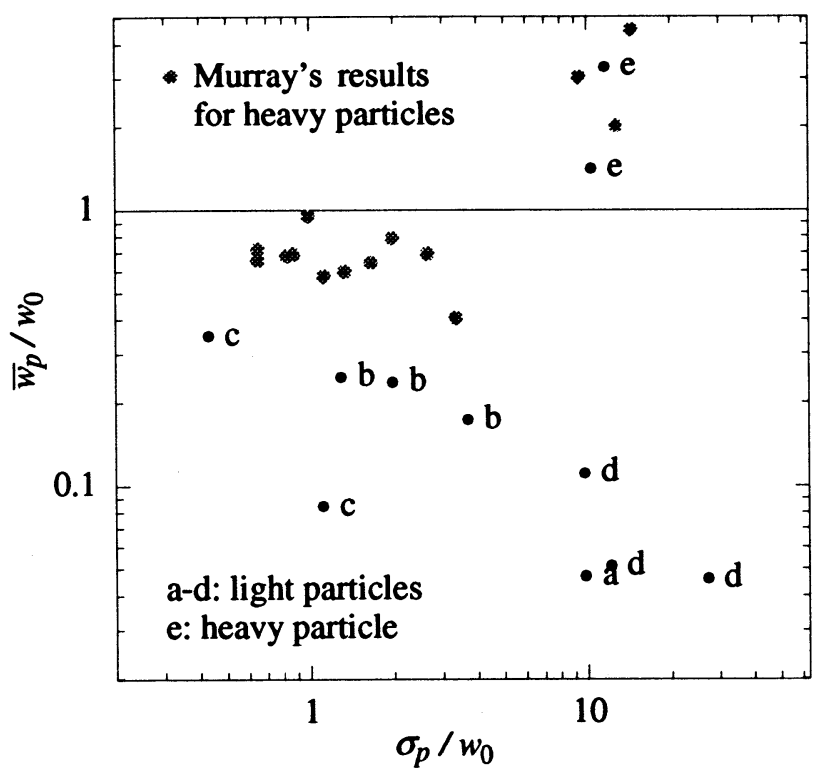

Fig. 1 Relative settling (or rising) velocity vs. relative turbulence intensity.

On the other hand, the light particles $\left(\rho_{p}<\right.$ $\left.\rho_{f}\right)$ are on average slowed down greatly in the range of $\sigma_{p} / w_{0}$ considered here. An decrease by a factor of 0.05 is observed. The decrease in the mean rising velocity of light particles can be mainly related to the phenomenon of 'trapping by vortices'. The reductions by vortex trapping are expected to be greater because light particles spiral inward, so that they can be trapped as long as the vertex lives. The light particles slow down as they are trapped by a vortex, move with it for a while, and then speed up again as the vortex disintegrates.

As shown in Fig.1, if the relative turbulence intensity is the same, the lighter particle (larger particle Reynolds number) indicate stronger decay of its rising velocity. In addition to the vortex trapping, there is a reduction induced by the lift force in the right-hand side of Eq. (9). The lift force causes the particle to move to the downflow region in the flow as illustrated by Fig. 2.

Small increases $(\leqslant 10 \%)$ in the settling velocity have been found in some numerical simulations ${ }^{8,9)}$. The inertial bias for a heavy particle makes the particle path along the periphery of the vortical structure as illustrated by Fig. 3. In strong turbulence, this fast-tracking effect can lead to very large increases in settling velocity because the asymptotic mean velocity along a fast track is proportional to turbulence intensity ${ }^{6}$.

\section{(2) Numerical simulations}

The turbulence intensity and life time of vortex have an influence on the settling or rising ve-

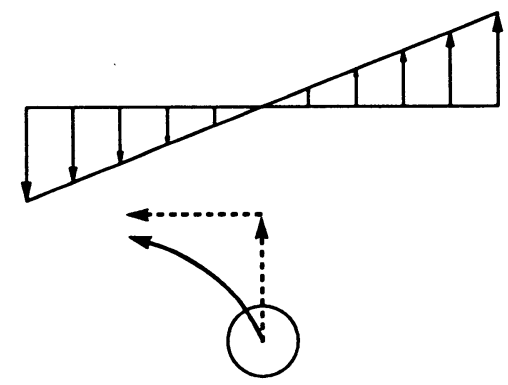

Fig. 2 Mechanism of reduction by the lift force.

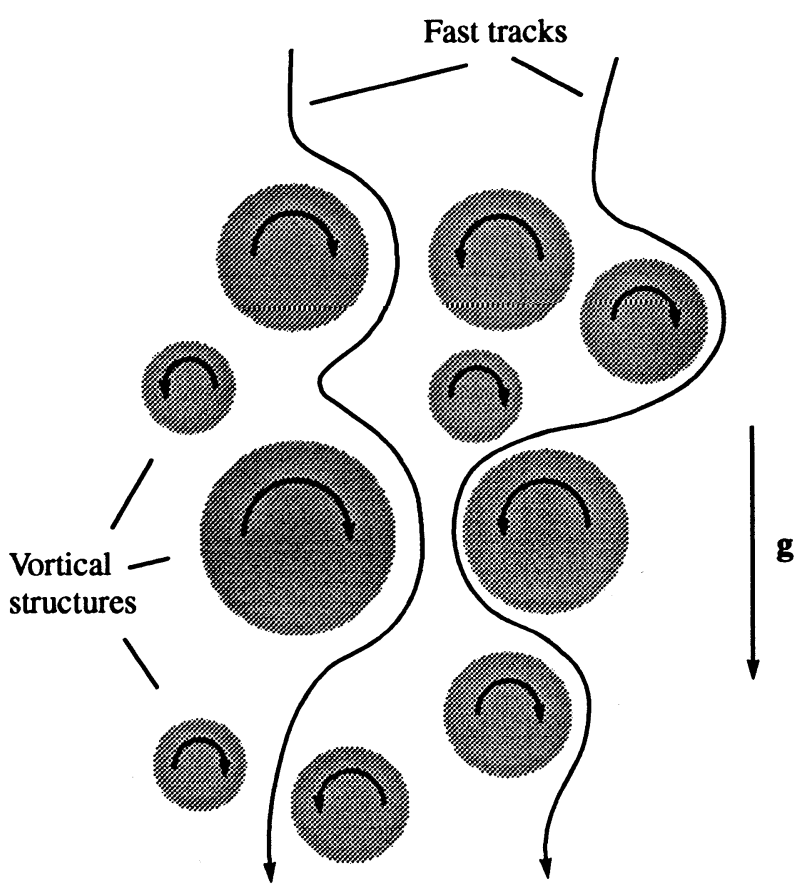

Fig. 3 Preferential sweeping (first tracking) mechanism for a heavy particle interacting with local flow vortical structures under its inertia and body force.

locity of a particle.

If the turbulence intensity is weak or if the vortices are short lived, the fast-tracking and trapping mechanisms of the vortices are not effective. In such cases a decrease rather than an increase in the settling velocity is caused by the turbulence.

The delaying effect may be explained in terms of a general loitering effect. The essence of the loitering effect is that a particle that is settling or rising through a nonuniform velocity field spends more time with fluid that moves opposite to its natural settling or rising velocity.

When the von Kármań-Pao spectrum is used, the Lagrangian integral time scale of fluid 


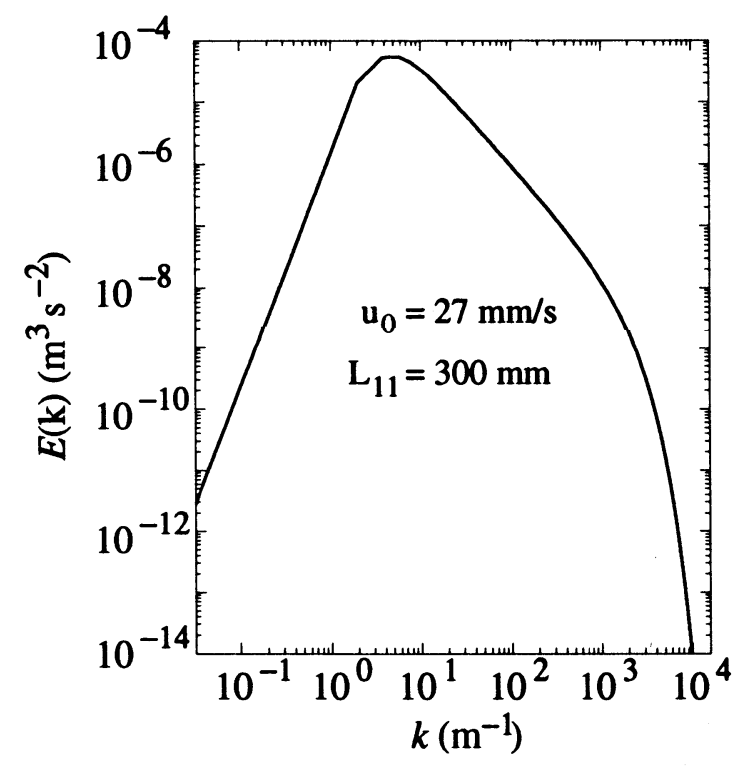

Fig. 4 Energy-spectrum function used in the numerical simulations.

particles satisfies the following relation ${ }^{11)}$ :

$$
T_{L}=\beta^{L} L_{11} / u_{0} ; \quad \beta^{L} \approx 0.5,
$$

which shows that, for a fixed value of the longitudinal integral scale, the Lagrangian integral time scale shortens in proportion to the turbulence intensity $u_{0}$. Thus, the mean life time of vortices is changed through the longitudinal integral scale and turbulent intensity. The von Kárman-Pao spectrum with $u_{0}=27 \mathrm{~mm} / \mathrm{s}$ and $L_{11}=300$ $\mathrm{mm}$ is shown in Fig. 4.

In the present simulation, we examine the settling or rising velocity of the same type of particles used in the experiments. We tentatively fix the longitudinal integral scale of turbulent field on $300 \mathrm{~mm}$. The results of simulations are shown in Fig. 5. The settling or rising velocity of particles is averaged over $20 T_{L}$ and over all (at least 200) realizations of the flow.

The results of simulations show the same trend as those in the experiments. The increases and reductions are, however, smaller than those in the experiments. The distinction between the experiments and the simulation may be attributed to the coherent structures of the turbulent velocity field. Unfortunately, since the velocity of fluid particle is not measured in the experiments, we cannot estimate the energy-spectra of fluid velocity and turbulence scales in the experiments. So, we cannot check their differences between turbulent fields in the experiments and those in the numerical simulations. However, it seems that the coherent structures of turbulence

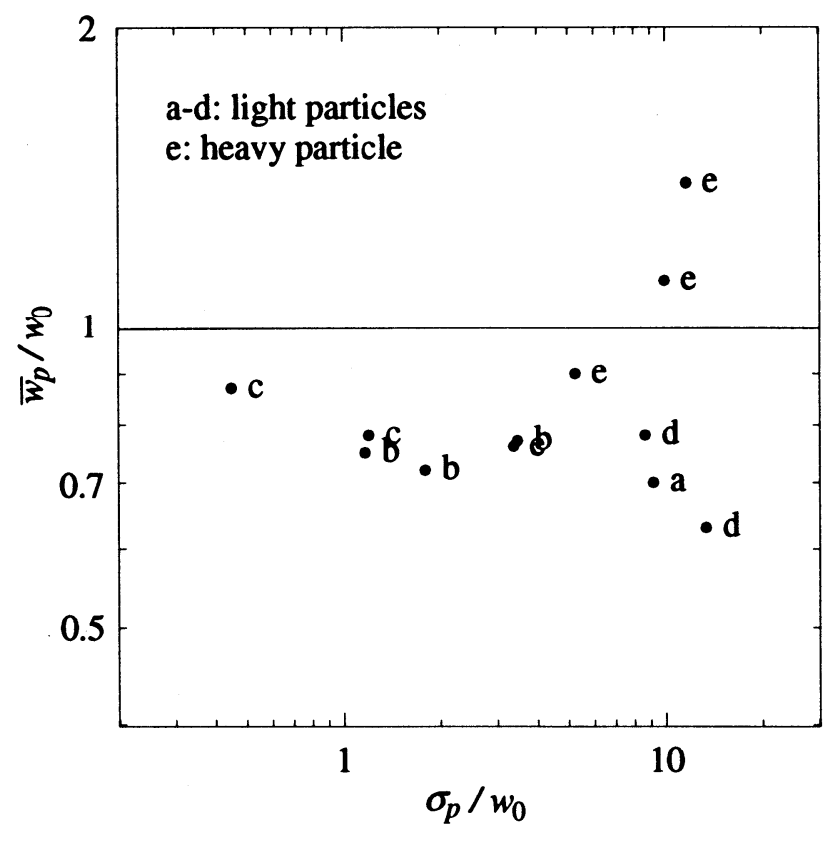

Fig. 5 Relative settling (or rising) velocity vs. relative turbulence intensity in the numerical simulations.

have stronger effects on the settling (or rising) velocity than the spectra.

Maxey $^{8)}$ used Kinematic Simulation (KS) with the Kraichnan spectrum function, which is representative for low Reynolds number turbulence behind a grid, to generate turbulent velocity fields, and then integrated an equation of motion of a heavy particle, subject to a Stokes drag force. Maxey's results ${ }^{8)}$ show that the particles are accelerated by the presence of the turbulent motion to a fall velocity that is on average at most $10 \%$ larger than in still fluid. Direct numerical simulations by Wang \& Maxey') show, however, that this increase in fall velocity can be much larger (up to $50 \%$ of the fall velocity in still fluid).

Wang \& Maxey $^{9)}$ pointed out that a basic distinction between their numerical simulation and that of Maxey ${ }^{8)}$ is that in Wang \& Maxey ${ }^{9}$ the turbulent flow evolves dynamically with the appropriate spatial and temporal structures, including the organized features of the dissipation range dynamics, while in the simulation technique (KS) used in Maxey ${ }^{8)}$ there was no such organization.

Moreover, Wang \& Maxey9) showed that heavy particles tend to move towards down-flow regions that lie around vortical zones in the turbulence. This preferential concentration of particles in these downflow regions leads to the acceleration of heavy particles by turbulent motion. 


\section{CONCLUSIONS}

The average scttling and rising velucily in homogeneous turbulent fields of a spherical particle have been examined by both experiments and numerical simulations. The settling velocity and rising velocity are slowed by $20-40 \%$ in relatively weak grid turbulence. The settling velocities are increased considerably in relatively strong turbulence. The experimental results show increases by up to a factor of 4 and the trend is towards greater increases for stronger turbulence. On the other hand, the light particles are slowed down greatly with increasing relative turbulence intensity. An decrease by a factor of 0.05 is observed for the light particles.

The results of simulations show the same trend as those in the experiments. However, the increases and reductions are smaller than those in the experiments. The distinction between the experiments and the numerical simulation may be attributed to the characteristics of turbulent velocity field: there are no organized structures in the turbulent field of the simulation.

The experimental data base urgently needs augmentation in various turbulent fields because different flow structures with the same relative turbulence intensity may have different effects on settling (or rising) velocity.

\section{REFERENCES}

1) Murray, S. P.: Settling velocities and vertical diffusion of particles in turbulent water, J. Geophys. Res., Vol. 75(March), pp. 1647-1654, 1970.

2) Nielsen, P.: Turbulence effects on the settling of suspended particles, J. Sedimen. Petrol., Vol.63(5), pp. 835-838, 1993.

3) Yamasaka, M. and Ikeda, S.: Theoretical study on mean fall velocity of spheres in fluids with vertical random oscillations, Proc. of JSCE, No.417/II-13, pp. 173-79, 1990 (in Japanese).

4) Gotoh, H., Tsujimoto, T. and Nkagawa, H.: Dynamics of suspended particle in vertical random oscillating flow, Proc. of Hydraulic Engrg., JSCE, Vol.38, pp. 585-90, 1994 (in Japanese).

5) Tooby, P. F., Gerald, L. W. and John D. I.: The mo- tion of a small sphere in a rotating velocity field: a possible mechanism for suspending particles in turbulence, J. Gcophys. Rcs., Vol.82(15), pp. 20962100, 1977.

6) Nielsen, P.: Coastal Bottom Boundary Layers and Sediment Transport, World Scientific, 1992.

7) Maxey, M. R. and Corrsin, S.: The gravitational settling of aerosol particles in randomly oriented cellular flow fields, J. Atmos. Sci., Vol.43, pp. 1112-1134, 1986.

8) Maxey, M. R.: The gravitational settling of aerosol particles in homogeneous turbulence and random flow fields, J. Fluid Mech., Vol.174, pp.441-465, 1987.

9) Wang, L. and Maxey, M. R.: Settling velocity and concentration distribution of heavy particles in homogeneous isotropic turbulence, J. Fluid Mech., Vol. 256, pp. 27-68, 1993.

10) Kraichnan, R. H.: Diffusion by a random velocity field, Phys. Fluids, Vol.13(1), pp. 22-31, 1970.

11) Fung, J. C. H., Hunt, J. C. R., Malik, N. A. and Perkins, R. J.: Kinematic simulation of homogeneous turbulence by unsteady random Fourier modes, $J$. Fluid Mech., Vol.236(10), pp. 281-318, 1992.

12) Hunt, J. C. R., Buell, J. C. and Wray, A. A.: Big whorls carry little whorls, Proc. of Summer Program, CTR, 1987.

13) Helland, K. N., Van Atta, C. W. and Stegen, G. R.: Spectral energy transfer in high Reynolds number turbulence, J. Fluid Mech., Vol.79, pp. 337-359, 1977.

14) Screenivasan, K. R.: On the scaling of the turbulence energy dissipation rate, Phys. Fluids, Vol.27, pp. 1048-1051, 1984.

15) Clift, R., Grace, J. R. and Weber, M. E.: Bubbles, Drops and Particles, Academic 1978.

16) Auton, T. R., Hunt, J. C. R. and Prud'homme, M.: The force exerted on a body in inviscid unsteady non-uniform rotational flow, J. Fluid Mech., Vol. 197, pp. 241-257, 1088.

17) Stoer, J. and Bulirsch, R.: Introduction to Numerical Analysis, Springer-Verlag, 1980.

(Received September 30,1998) 